\title{
Simulation of CGW Injection in Parts of a Fluvial Deposited Reservoir
}

\author{
Slotte P. A., Solheim A., Jensen J. I.
}

IKU, Norway

\begin{abstract}
Copyright 1995, Steering Committee of the European IOR - Symposium.
This paper was presented at the 8th. European IOR - Symposium in Vienna, Austria, May 16 - 17, 1995

This paper was selected for presentation by the Steering Committee, following review of information contained in an abstract

submitted by the author(8). The paper, as presented has not been reviewed by the Steering Committee.
\end{abstract}

\begin{abstract}
Poor sweep efficiency and early break through is a main concern with respect to improved oil recovery by gas injection processes. These adverse effects may be eliminated by combined gas and water (CGW) injection. The efficiency of a CGW injection process is strongly related to the size of the mixed gas/water flow zone in the reservoir.

The work presented here deals with reservoir heterogeneities and geometry within a single conducting channel in a fluvial deposited reservoir.

Heterogeneities in terms of variable permeability was generated by stochastic simulation. These permeabilities were furthermore modified to study the effect of different heterogeneity profiles in terms of average permeability versus depth, and of heterogeneity contrast in terms of the spread of permeability around this average value.

The importance of compositional effects, was studied.

The main conclusions are:

1. Larger heterogeneity contrast extends the mixed flow zone.

2. Within a conducting channel, the mixed zone is larger than what may be derived from Stone's segregation model. This is due to capillary effects and mixing due to heterogeneity contrast.
\end{abstract}

3. Stone's model is, however, a suitable tool for a rough estimation of the size of the mixed zone, both for simultaneous and alternating gas and water injection.

4. Strong improvement can be obtained by using CGW injection compared with pure gas injection.

\section{Introduction}

Poor sweep efficiency and early break through is a main concern with respect to improved oil recovery by gas flooding in general.

The sweep efficiency may be improved in combined gas/water (CGW) injection processes through reduced gas mobility. The efficiency of a CGW injection process is strongly related to the size of the mixed gas/water flow zone in the reservoir. The fractional size of this mixed zone with respect to the flooded reservoir volume, depends on reservoir parameters such as the length/height ratio of the reservoir segment in question, the reservoir heterogeneities at different scales, capillary pressure and the dynamic reservoir parameters.

The present paper deals with the effect of reservoir heterogeneities within a fraction of a fluvial deposited reservoir. Heterogeneities in terms of variations in permeability was generated through 
stochastic simulation. These heterogeneities were furthermore modified to study the effect of different kinds of heterogeneity profiles in terms of average permeability in each layer versus depth, and to study the effect of varying heterogeneity contrast in terms of variation around a mean value within each layer.

The main simulations were run on the black oil simulator SIMBEST II $/ 1 /$, while the studies of compositional effects were run on ECLIPSE 300 $/ 2 /$ in black oil and compositional mode.

\section{Reservoir model}

The selected model is based on reservoir and geological data from a fluvial deposited North Sea reservoir. These data formed the basis for stochastic reservoir simulation. Two realizations were used.

A small section of the model, representing a single conducting channel within the fluvial system was used in the simulations.

The grid model consists of $25 \times 8 \times 20$ blocks, covering an area of $1825 \mathrm{~m} \times 1260 \mathrm{~m}$ with an average thickness of $6 \mathrm{~m}$. The model has an overall dip of $3.5^{\circ}$, and the injector is located updip. Both wells are perforated in all layers.

Seven different heterogeneity patterns within the channel was generated by modifying the original stochastically generated realization:

- Equally distributed heterogeneities (average $200 \mathrm{mD}$ ), low (original) contrast.

- Equally distributed heterogeneities, high contrast.

- Upwards increasing sequence (average from $100 \mathrm{mD}$ to $2000 \mathrm{mD}$ varying linearly on logarithmic scale), low (original) contrast.

- Upwards increasing sequence, high contrast.

- Downwards increasing sequence, low (original) contrast.

- Thief zone on top (average $3000 \mathrm{mD}$, rest with average $200 \mathrm{mD}$ ), low (original) contrast.

- Thief zone on bottom, low (original) contrast.

The basic reservoir data are shown in Table 1.

\section{Table 1 Basic reservoir data.}

\begin{tabular}{|l|c|}
\hline Initial reservoir pressure & 382 bar \\
Reservoir temperature & $100^{\circ} \mathrm{C}$ \\
Reservoir depth & $2475 \mathrm{~m}$ \\
Water oil contact depth & $2561 \mathrm{~m}$ \\
Initial gas oil ratio & 121 \\
\hline
\end{tabular}

\section{Generating heterogeneity profiles}

The heterogeneity profiles were generated as outlined below:

First, the geometric average horizontal permeability for each layer in the original realization was calculated for $x$ and $y$ directions separately:

$$
\bar{k}_{x k}^{o}=k_{0} \exp \left(\frac{1}{N_{x} N_{y}-q} \sum_{i, j} \ln \left(k_{x i j k}^{o} / k_{0}\right)\right) \text {. }
$$

Only active blocks are included in the summation, and blocks with a permeability less than $0.1 \mathrm{mD}$ are considered inactive. The average is calculated separately for the permeability in $x$ and $y$ direction.

The horizontal permeability for each block for the desired heterogeneity profile is calculated using the following formulation:

$k_{x i j k}^{n}=\left[\frac{k_{x i j k}^{o}}{\bar{k}_{x k}^{o}}\right]^{b} \cdot \bar{k}_{x k}^{n}$,

where the superscript $n$ indicate the desired heterogeneity profile. The exponent $b$ is included to be able to adjust the heterogeneity contrast. For $b=1$, the original contrast is retained, while an exponent $b>1$ generate a higher contrast, and $b<1$ a lower contrast.

The permeability in the $x$ and $y$ direction is calculated separately by the same formula (by exchanging the subscript $x$ with $y$ ).

New vertical permeabilities are calculated using the following formulation:

$k_{z i j k}^{n}=\left[\frac{k_{z i j k}^{o} \cdot k_{z i j k}^{o}}{\bar{k}_{x k}^{o} \cdot \bar{k}_{y k}^{o}}\right]^{b / 2} \cdot \bar{k}_{x k}^{n}$.

\section{Relative permeability and capillary pressure}

When a reservoir section is subjected to WAG injection, the water saturation in parts of the section will oscillate over time. The water saturation will increase as a result of a water injection cycle, and decrease following a gas injection cycle. This implies that the effect of relative permeability and capillary pressure hysteresis must be included in a simulation study. To model hysteresis in the simulators, two sets of relative permeability and capillary pressure curves must be supplied: Drainage curves, applicable when the water saturation is decreasing, and imbibition curves, applicable when the water 
saturation is increasing. When the saturation change in a particular grid block changes direction, a scanning curve running between the two pure curves is traced.

In a WAG situation, gas injection will be related to the drainage process and water injection to the imbibition process.

The simulators that have been used employ different hysteresis models. Care has been taken to give input data that give as close results as possible.

In a heterogeneous reservoir; the permeability variations will be accompanied by variations in relative permeability and capillary pressure. It was decided to model this by defining five different rock types and assigning grid blocks to rock type according to the criteria given in Table 2 . Within each permeability interval, the relative drainage capillary pressure curves used is of Corey type $/ 3,4 /$ :

$P_{\mathrm{c}}=P_{\mathrm{e}}\left(\frac{S_{\mathrm{w}}-S_{\mathrm{wi}}}{1-S_{\mathrm{wi}}}\right)^{-\frac{1}{\lambda}}$.

The drainage relative permeabilities are based on the same formalism:

$$
\begin{aligned}
k_{\mathrm{rw}}^{\mathrm{dr}}= & \left(\frac{S_{\mathrm{w}}-S_{\mathrm{wi}}}{1-S_{\mathrm{wi}}}\right)^{\frac{2+3 \lambda}{\lambda}}, \\
k_{\mathrm{row}}^{\mathrm{dr}}= & k_{\mathrm{r}}^{\mathrm{o}} \cdot\left(\frac{S_{\mathrm{o}}}{1-S_{\mathrm{wi}}}\right)^{2} \cdot \\
& {\left[\begin{array}{l}
1-\left(\frac{1-S_{\mathrm{o}}-S_{\mathrm{wi}}}{1-S_{\mathrm{wi}}}\right)^{\frac{2+\lambda}{\lambda}} \\
k_{\mathrm{rog}}^{\mathrm{dr}}=
\end{array}\right] } \\
& {\left[\begin{array}{l}
\mathrm{o}\left(\frac{S_{\mathrm{o}}-S_{\mathrm{org}}}{1-S_{\mathrm{wi}}-S_{\mathrm{org}}}\right)^{2} . \\
1-\left(\frac{S_{\mathrm{o}}-S_{\mathrm{org}}}{1-S_{\mathrm{wi}}-S_{\mathrm{org}}}\right)^{\frac{2+\lambda}{\lambda}}
\end{array}\right], }
\end{aligned}
$$

and

$$
\begin{aligned}
k_{\mathrm{rg}}^{\mathrm{dr}}= & k_{r}^{o} \cdot\left(\frac{S_{\mathrm{g}}}{1-S_{\mathrm{wi}}}\right)^{2} . \\
& {\left[1-\left(\frac{1-S_{\mathrm{g}}-S_{\mathrm{wi}}}{1-S_{\mathrm{wi}}}\right)^{\frac{2+\lambda}{\lambda}}\right] }
\end{aligned}
$$

Imbibition relative permeabilities and capillary pressure curves have been chosen such that they follow the drainage curves at high saturations. However, at a selected point $\left(S_{\mathrm{d}}=0.4\right)$ they start to deviate so that they end in the appropriate end points, $S_{\text {orw }}$ and $S_{\mathrm{gr}}$. This is obtained by using the urainage curve, and applying a linear scaling of the saturations over the interval between $S_{\mathrm{d}}$ and the end point.

The relative permeability parameters used for each of the five rock types are given in Table 2 .

Table 2 Relation between permeability and the parameters determining the relative permeabilities and the capillary pressure data.

\begin{tabular}{|c|c|c|c|c|}
\hline $\begin{array}{c}\text { Abs.per } \\
\mathrm{m} .(\mathrm{mD})\end{array}$ & $\begin{array}{c}\text { Poros. } \\
\text { (frac) }\end{array}$ & $\lambda$ & $S_{\mathrm{wi}}$ & $S_{\text {orw }}$ \\
\hline$<40$ & 0.14 & 1.0 & 0.35 & 0.10 \\
\hline $40-200$ & 0.17 & 2.5 & 0.30 & 0.15 \\
\hline $\begin{array}{c}200- \\
1000\end{array}$ & 0.20 & 3.0 & 0.25 & 0.20 \\
\hline $\begin{array}{c}1000- \\
4000\end{array}$ & 0.23 & 3.5 & 0.20 & 0.25 \\
\hline$>4000$ & 0.264 & 4.0 & 0.10 & 0.30 \\
\hline $\begin{array}{c}\text { Abs.per } \\
\mathrm{m} .(\mathrm{mD})\end{array}$ & $S_{\text {org }}$ & $S_{\mathrm{gr}}$ & $p_{\mathrm{ce}}$ & $k_{r}^{o}$ \\
\hline$<40$ & 0.05 & 0.05 & 0.06 & 0.65 \\
\hline $40-200$ & 0.07 & 0.06 & 0.05 & 0.70 \\
\hline $\begin{array}{c}200- \\
1000\end{array}$ & 0.10 & 0.07 & 0.04 & 0.76 \\
\hline $\begin{array}{c}1000- \\
4000\end{array}$ & 0.12 & 0.08 & 0.03 & 0.80 \\
\hline$>4000$ & 0.15 & 0.10 & 0.01 & 0.85 \\
\hline
\end{tabular}

The end point saturations for oil and gas are selected to be highest in the high permeability zones and lowest in the low permeability zones.

In the simulators, three phase relative permeability are calculated on the basis of two phase data. The simulated efficiency of a WAG process may depend on the choice of three phase relative 
permeability correlation. In SIMBEST II, a modification of a Dietrich-Bonder equation /1,5/ was employed: . :

$k_{r o}=k_{r}^{o}\left[\left(\frac{k_{\text {row }}}{k_{r o}}+k_{r w}\right)\left(\frac{k_{r o g}}{k_{r}^{o}}+k_{r g}\right)-k_{r g}-k_{r w}\right]$.

In ECLIPSE, the eclipse default model / $/ 2$ was used:

$k_{\mathrm{ro}}=\frac{S_{\mathrm{g}} k_{\mathrm{rog}}+\left(S_{\mathrm{w}}-S_{\mathrm{wi}}\right) k_{\mathrm{row}}}{S_{\mathrm{g}}+S_{\mathrm{w}}-S_{\mathrm{wi}}}$.

\section{Fluid description}

The pVT data used in the main part of the study, employ live oil $\left(R_{\mathrm{s}} \neq 0\right)$ and dead gas $\left(R_{\mathrm{v}}=0\right)$, and are relevant for injection of equilibrium gas. For the study of compositional effects, three new pVT models were generated: An 8 component compositional model, and two black oil models. The black oil models were intended to reproduce the phase behavior of the compositional model as closely as possible. One of the models is relevant for injection of equilibrium gas, while the other model is relevant for injection of separator gas. Both new black oil models include live oil $\left(R_{\mathrm{s}} \neq 0\right)$ and live gas $\left(R_{\mathrm{v}} \neq 0\right)$.

\section{Compositional model}

The Intera PVT program /6/ was used to tune an eight component compositional model employing the SRK equation of state with a volume shift parameter. The main goal in the tuning process was to create a compositional model that reproduce the black oil data of the original model as closely as possible.

\section{Black oil models}

The compositional model was used as a basis for generating the new black oil models with live oil $\left(R_{\mathrm{s}} \neq 0\right)$ and live gas $\left(R_{v} \neq 0\right)$. The Intera PVT program was employed for this. Black oil tables generated by this program are independent of the injection gas used, and are mainly relevant for depletion processes or injection of equilibrium gas. An alternative algorithm for generating black oil data more relevant for gas injection processes, with respect to phase behavior, was used.



Figure 1 Phase diagram of a two component system (oil and gas), and method for generating black oil data intended to represent gas injection processes.

The method is illustrated in Figure 1: Depending on pressure and the relative amounts of initial undersaturated reservoir oil and injection gas, the reservoir hydrocarbon system will exist in one of four states: Gas, oil, gas and oil at coexistence, or supercritical. The initial reservoir fluid (1) contains some dissolved gas (initial $R_{\mathrm{s}}$ ). When the pressure is reduced, the coexistence line is crossed at the initial bubble point pressure (2), and the equilibrium gas (3) contains some dissolved oil (initial $R_{\mathrm{v}}$ ). The density of coexisting oil and gas define $B_{\mathrm{o}}$ and $B_{\mathrm{g}}$ at the given (bubble point) pressure. Additional points on the coexistence curve are found from the properties of mixtures of reservoir fluid and injection gas: Each mixture (4) contains an increased amount of dissolved gas, and the coexistence line is crossed at a higher bubble point pressure (5). The new equilibrium gas (6) typically contain an increased amount of dissolved oil. The density of coexisting oil and gas define $B_{\mathrm{o}}$ and $B_{\mathrm{g}}$ at the new bubble point pressure. Note that, due to the changed surface density, the $B_{\mathrm{o}}$ and $B_{\mathrm{g}}$ values of the mixture must be adjusted so that the correct reservoir densities are reproduced:

$$
\begin{aligned}
& B_{\mathrm{o}}=\left(\frac{\rho_{\mathrm{os}}+R_{\mathrm{s}} \rho_{\mathrm{gs}}}{\rho_{\mathrm{os}}^{\prime}+R_{\mathrm{s}} \rho_{\mathrm{gs}}^{\prime}}\right) B_{\mathrm{o}}^{\prime} \\
& B_{\mathrm{g}}=\left(\frac{\rho_{\mathrm{gs}}+R_{\mathrm{v}} \rho_{\mathrm{os}}}{\rho_{\mathrm{gs}}^{\prime}+R_{\mathrm{v}} \rho_{\mathrm{os}}^{\prime}}\right) B_{\mathrm{g}}^{\prime}
\end{aligned}
$$

The difference between the three black oil models are illustrated in Figure 2. The original model and the equilibrium gas model are very similar up to a pressure of $p \approx 300 \mathrm{bar}$, where vaporization becomes important. The separator gas swells more oil, and is miscible above $p \approx 300$ bar. Note that above this pressure, the method outlined above gives a two phase zone of 
basically zero thickness. The finite thickness shown in Figure 2 was obtained by arbitrarily lifting the gas coexistence (i.e. $R_{v}$ ) line to avoid numerical problems resulting from an instant jump from $100 \%$ oil to $100 \%$ gas.

\section{Simulation results}

Simulation constraints were selected such that the results would be less sensitive to other variables than heterogeneities and geometry. An updip injection was therefore used so that the draw-back of high gas mobility compared to water was compensated by gravity advantages.

The water/gas ratio was chosen equal to one. This gives breakthrough for water and gas at approximately the same time in the base case.

The WAG cycle length was 200 days starting with 100 days of water injection.

The injection rate is chosen in order to flood the reservoir segment with one pore volume in 4500 days, corresponding to $500 \mathrm{Rm}^{2} /$ day for the base case. Due to the choice of porosity versus permeability, the pore volumes are different in the different cases. Keeping the average interstitial velocity constant, the injection rates were varied from $453 \mathrm{Rm}^{3} / \mathrm{d}$ to $532 \mathrm{Rm}^{3} / \mathrm{d}$ for the heterogeneity cases. For the cases where the thickness was increased, the rate was correspondingly increased in order to keep the interstitial velocity constant. Initially there was no gas cap.

\section{Gas and water breakthrough}

Early breakthrough of injection fluids will normally lead to reduced oil rates, and low sweep efficiency

One idea behind a WAG injection is to delay the breakthrough time, by controlling the mobility of the injected gas.

The results, related to breakthrough time of gas and water in the heterogeneous cases, are given in Figure 3. There is a significant difference between the heterogeneity profiles, and a small difference between the two realizations. However the tendency within each realization is the same.
Hence the conclusions are not sensitive to the choice of realization.

Gas is the breakthrough fluid for all the heterogeneous profiles except for downwards increasing and thief zone on bottom profiles. These two profiles are less favorable for gas override so a later gas breakthrough in these cases should be expected.



Figure 2 Phase diagrams corresponding to the three different black oil models. The original dead gas model (a), the equilibrium gas model with live gas (b), and the separator gas model (c). 


\section{Gas Breakthrough Time}



Water Breakthrough Time



Figure 3 Gas and water breakthrough times, in units of pore volume injected, for the different heterogeneity profiles and realizations.

Comparing the cases of low and high permeability contrast the water breakthrough is earlier for the case having high contrast. For gas breakthrough this is only true for the equally distributed profile. The results are opposite for the upwards increasing sequence. A larger contrast will promote the mixing of phases and thus postpone the breakthrough. The creation high conductivity channels by heterogeneity induced viscous fingers will, on the other hand, also be promoted. In the upwards increasing profile the gas will have a stronger tendency for overriding. The channeling mechanism should thus be stronger in the top layers promoting an earlier breakthrough of gas. However, the opposite is observed indicating that the mixing promotion is dominating.

\section{Mixed zone sweep efficiency}

For combined gas/water injection, Mobility control of the injected fluid(s) can only take place in the mixed flow zone.

The mixed volumetric sweep is defined as the fraction of the reservoir blocks where the saturation of both gas and water has increased by a fraction of at least 0.05 at any time during the simulation. Results from the simulations are shown in Figure 4. There is a significant difference in mixed volumetric sweep between the different heterogeneity profiles.

The heterogeneity contrast seem to have a uniform influence on the size of the mixing zone.

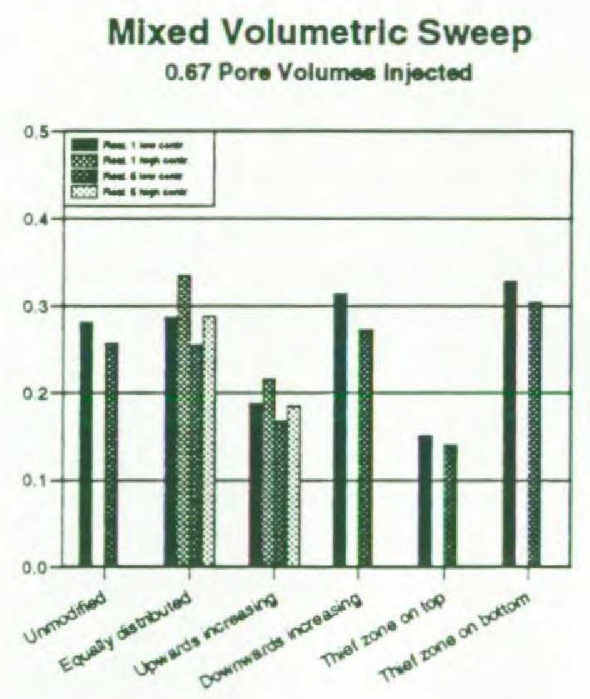

Figure 4 Mixed volumetric sweep for the different heterogeneities and realizations.

A larger mixed zone will delay the breakthrough of the injected fluid and increase the recovery. The larger mixing zone correlates well to the data of breakthrough.

An other important aspect is the location and saturation of the mixed zone. Figure 5 shows the location and saturation change in the mixed zone for heterogeneity profiles of "equally distributed," "upwards increasing," and "downwards increasing" of Realization 5 for a selected $x-z$ cross section running through the injection well $(j=4)$. 




For the upwards increasing sequence (Figure 5, lower left) the zone swept by gas is identical to the mixed zone in the selected cross section. There is no gas zone, only the mixed zone above the gas/water contact. However, on this particular case with very low permeability at the bottom layers, the simulator closes the well in these bottom layers during gas injection in order to avoid back flow.

The situation for the equally distributed (upper left) sequence, a very thin gas zone can be observed on the top. The vertical size of the mixed zone is increased in relation to the upwards increasing sequence.

For the downwards increasing sequence (upper right) the vertical size of the free gas and mixed zone is increased.

Common for all of the cases is that the gas segregation do not go further than a distance of about 400 meters from the injection well. This distance is approximately 0.3 of the distance between the wells and can be compared directly to the viscous to gravity ratio, VGR, in the segregation model of Stone. ${ }^{3}$ For the current cases VGR is estimated to about 0.01 , which in the near well region, where the flow is approximately radial, corresponds to a relative distance of $\sqrt{\mathrm{VGR}} \approx 0.1$. The comparison with the theory is elaborated upon in Section 0 below.

Another aspect in the situation after the stabilization of the segregation at $x>0.30$, is the thickness of the mixed zone. The thickness of the mixed zone will correspond to a transition zone related to the capillary pressure curve. As long as the gas velocity is small enough, the process will be controlled by capillary equilibrium $\left(P_{\mathrm{c}}=\Delta \rho g h\right)$. This effect is confirmed in a run where capillary pressure was reduced by two orders of magnitude. The thickness the transition zone is then reduced to slightly more than a single block as determined by numerical dispersion.

\section{Analytical theory for the size of the mixed zone}

Analytical expressions for the shape of the mixed zone are given by Stone $/ 7 /$ and Jenkins /8/. These expressions are valid under the following conditions:

- Simultaneous injection of water by miscible gas, at rates constant in time and along the injection well.

- Homogeneous non dipping reservoir of constant thickness.

- Steady state.

- Zero capillary pressure.

- Zero compressibility. 
The theory may be extended to immiscible displacement by noting that the oil saturation reachès the end point values $S_{\text {org }}$ and $S_{\text {orw }}$ after gas and water flooding, respectively. Similarly an end saturation of $S_{\text {orm }}$ is reached after mixed flooding.

In the present simulations the water and gas injection is alternating. . This implies that steady state conditions are never reached. The size and location of the mixed zone will change during the injection cycle. The zone reaches its maximum in the near well region at the end of gas injection, the maximum point, 0.67 pore volumes injected, has been used here.

In the near well region we may assume radial displacement and the height of the water zone is then given by

$h_{\mathrm{w}}(r)=\frac{M_{\mathrm{gw}} \mathrm{WAG}}{\alpha+M_{\mathrm{gm}} \operatorname{VGR}\left(R^{2} / r^{2}\right)} h$,

with

$\alpha=1+M_{\mathrm{gw}} \mathrm{WAG}-M_{\mathrm{gm}}$.
WAG is the WAG ratio, i.e. the volumetric ratio of injected water to gas, and VGR is the viscous to gravity ratio defined by

$\mathrm{VGR}=\frac{q_{\mathrm{t}}}{\Delta \mathrm{p} \cdot k_{\mathrm{v}} g \cdot a\left(\lambda_{\mathrm{gm}}+\lambda_{\mathrm{wm}}\right)}$.

$M_{\mathrm{gw}}$ and $M_{\mathrm{gm}}$ are mobility ratios:

$M_{\mathrm{gw}}=\frac{\lambda_{\mathrm{gg}}}{\lambda_{\mathrm{ww}}}$, and $M_{\mathrm{gm}}=\frac{\lambda_{\mathrm{gg}}}{\lambda_{\mathrm{gm}}}$.

VGR has been calculated for a semicircle of radius $R=\frac{1}{2} L_{y}$, where $L_{y}$ is the

width of the reservoir, and a sweep efficiency of $80 \%$.

The theoretical predictions, for a flat homogeneous reservoir of height $6 \mathrm{~m}$ and ignoring the small dip $\left(\approx 3^{\circ}\right)$, are compared to simulation results in Figure 6.

In the simulations, the mixed zone boundary is defined as the $10 \%$ change level in the simulations (Figure 5).


Figure 6 Extension of the mixed zone in $x z$ cross section $(j=4)$ for different geometries: Unmodified (upper left), double height (upper right), four times the height (lower left), and half length (lower right). Simulated results are compared with the theory of Jenkins 18 . 
Considering all the non valid approximations, the analytical theory performs surprisingly well in giving estimates for the size of the mixed zone.

\section{Compositional effects}

The equally distributed, upwards increasing, and downwards increasing heterogeneity distributions based on one of the realizations, were simulated using the different fluid descriptions described in section 0 to evaluate compositional effects: Compositional simulations using equilibrium gas and separator gas as injection gas, and the two new black oil models employing live gas.

The break trough times, size of mixed zone, and recovery is heavily dependent on the fluid description. However, the main conclusions as stated in section 0 below remain valid independent of fluid description.

\section{Conclusions}

The results from the simulations of the current reservoir segment using varying "heterogeneity profiles" have lead to the following conclusions:

1) Heterogeneity contrasts extend the gas/water mixed zone.

2) Mixed zone within thin conducting channels is greater than what may be derived from Stone's segregation model.

3) The segregation model of Stone seem to be good enough for a rough estimation of the size of the mixing zone.

4) Simulations based on the two realizations show the same trends in the simulated results.

5) Strong improvement can be obtained by using up-dip WAG injection instead of pure gas in thin reservoirs with dip.

6) Within a certain maximum channel thickness, the thickness of the channel seems to be less important.

\section{Nomenclature}

\section{Symbols}

$$
\begin{array}{ll}
\mathrm{a} & =\text { Swept area } \\
\mathrm{b} & =\text { Heterogeneity index } \\
\mathrm{B} & =\text { Formation volume factor (subscript for } \\
& \text { phase) } \\
\mathrm{g} & =\text { Acceleration of gravity } \\
\mathrm{hw}(\mathrm{r}) & =\text { Height of water zone in radial model } \\
\mathrm{k} & =\text { Permeability }
\end{array}
$$

\begin{tabular}{|c|c|c|}
\hline 'g & $=$ & Gas \\
\hline gg & $=$ & Gas in gas zone \\
\hline $\operatorname{gm}$ & $=$ & Gas in mixed zone \\
\hline gr & $=$ & Residual gas \\
\hline $\mathbf{L}$ & $=$ & Liquid \\
\hline 0 & $=$ & Oil \\
\hline org & $=$ & Residual oil in oil-gas system \\
\hline orm & $=$ & Residual oil in mixed zone \\
\hline orw & $=$ & Residual oil in oil-water system \\
\hline rg & $=$ & Relative to gas \\
\hline ro & $=$ & Relative to oil \\
\hline row & $=$ & Relative to oil in water oil system \\
\hline rw & $=$ & Relative to water \\
\hline $\operatorname{rog}$ & $=$ & Relative to oil in gas oil system \\
\hline $\mathrm{t}$ & $=$ & Total \\
\hline $\mathbf{v}$ & $=$ & Vertical \\
\hline $\mathbf{w}$ & $=$ & Water \\
\hline wi & $=$ & Irreducible water \\
\hline wm & $=$ & Water in mixed zone \\
\hline ww & $=$ & Water in water zone \\
\hline wt & & Irreducible wetting phase \\
\hline xijk & $=$ & $\mathrm{X}$-direction in block ijk \\
\hline $\mathbf{x k}$ & & $\mathrm{X}$-direction in layer $\mathbf{k}$ \\
\hline zijk & & Z-direction in block ijk \\
\hline zk & $=$ & Z-direction in layer $\mathbf{k}$ \\
\hline 0 & $=$ & Reference \\
\hline
\end{tabular}



\section{Subscript}




\section{Superscript}

$$
\begin{array}{ll}
\mathrm{dr} & =\text { Drainage } \\
\mathrm{o} & =\text { Reference } \\
* & =\text { Effective } \\
\mathrm{n} & =\text { New modified / desired }
\end{array}
$$

Abbreviations etc.

pVT = Pressure-volume-temperature

CGW = Combined gas water injection

WAG = Water alternating gas injection

\section{References}

/1/ Scientific Software Intercomp Inc.: SIMBEST II, Version 3.0, Users manual. (Dec. 1990).

12 Intera Information Technologies: Eclipse 300 Technical description version 94a, Henley-on-Thames, England 1994.

13/ BROOKS,R.H.and COREY, A.T.: Hydraulic Properties of Porous Media. Hydraulic Paper Number 3, Colorado State University, 1964.

14/ BROOKS,R.H., and COREY, A.T.: Properties of Porous Media Affecting Fluid Flow. Journal of the Irrigation and Drainage Division Proc. of ASCE (1968), vol.92, No IR2, pp. 61-88.

15/ DIETRICH,J.K.and BONDER,P.L.:Threephase relative permeability models. SPE 6044 presented at the 51st annual fall meeting of SPE of AIME (Oct 1976).

16/ Intera Information Technologies: PVT Reference manual version 94a, Henley-onThames, England 1994.

171 STONE, H.L.: Vertical Conformance in an Alternating Water-Miscible Gas Flood, Paper SPE 11130, presented at the SPE 57th annual fall technical conference and exhibition, New Orleans (Sep. 1982).

18/ JENKINS, M.K.: An Analytical Model for Water/Gas Miscible Displacements. Paper SPE 12632 presented at the SPE/DOE Fourth Symposium on Enhanced Oil Recovery, Tulsa (April 1984).

\section{Acknowledgment}

The RUTH Combined Gas/Water Injection Sub-program was initiated by the Norwegian authorities and is financially supported by the Research Council of Norway. Additional to this, the program is supported by technical assistance, quality control and finance through four years by the following oil companies: Amoco Norway, Conoco Norway, Elf Petroleum Norway, Enterprise Oil, Idemitsu, Neste Petroleum, Norsk Agip, Norsk Hydro, Phillips Petroleum Norway, Saga Petroleum, Svenska Petroleum and Total Norge. Their support is gratefully acknowledged. 